\title{
PDMS-BASED NANOIMPRINT LITHOGRAPHY FOR PHOTONICS
}

\begin{abstract}
Implementation of planar surface photonic crystal $(P h C)$ structures leads to improvement of optical properties of optoelectronic devices. Application of such structures can be attractive for overall and local enhancement of light from patterned areas of the light emitting diode surface and for lightwave-guiding devices. We present techniques useful for PhC patterning and for patterning of different optic structures in surface of new promising material polydimethylsiloxane (PDMS). Paper proposes nanoimprint technology for fabrication of PhC in the surface of thin PDMS membranes for possible application in light emitting diodes. By PDMS patterning and its positioning on the device surface one can achieve original optical properties of optoelectronic devices. We also present new technique for fabrication of surface-relief fiber Bragg grating (SR FBG) based on PDMS waveguide.
\end{abstract}

Keywords: Polydimethysiloxane, nanoimprint lithography, photonic crystal.

\section{Introduction}

Photonics has recently become an intensively evolving area where the possibilities of photonic structures or crystals $(\mathrm{PhC})$ for integrated optoelectronics were found. PhCs have a periodic change of refractive index at the interface of two materials, which causes the creation of photonic band gap in the crystal [1 and 2]. These features of PhCs cause the effects that are not possible to obtain by means of conventional optical and optoelectronic devices. From the application point of view, there is a wide range of $\mathrm{PhCs}$ applications with perspective of significant improvement of features of optoelectronic devices as light emitting diodes, lasers and optical waveguides with photonic structure [3 - 5]. Typically, a photonic light emitting diode (LED) shows enhancement of light extraction efficiency due to the improvement of total internal reflection on the semiconductor-air interface diffraction light on surface roughness or photonic band gap [6]. Also $\mathrm{PhC}$ in lightwave-guiding applications increased the transmission of sharp bended waveguides and Y-splitters [7].

There are different ways how to fabricate optical elements and devices with patterned surface for application in optics and optoelectronics. This paper summarizes few techniques useful for $\mathrm{PhC}$ patterning and for patterning of different optic structures using new promising material polydimethylsiloxane (PDMS), with significant optic and elastic properties. In visible range of spectrum, PDMS shows high transparency and is well formable by imprinting techniques. By PDMS patterning and its positioning on the device surface one can achieve original optical properties of optoelectronic devices [8]. The paper proposes technology for fabrication of PDMS membranes which were patterned by $\mathrm{PhC}$ in the surface. Such PhC PDMS membranes can be attractive for application in optoelectronic devices. Using direct laser writing (DLW) technique, Fresnel zone plate (FZP) was prepared in the surface of thin PDMS membrane. We also present a new technique for fabrication of surface-relief fiber Bragg grating (SR FBG) embedded in PDMS waveguide.

\section{PDMS membranes with PHC}

Typically, $\mathrm{PhC}$ are prepared on different metal and semiconductor surfaces. Here, we present a technique for surface patterning of thin PDMS membranes. In this experiment we used interference lithography in combination with PDMS imprinting to prepare planar two-dimensional (2D) $\mathrm{PhC}$ at the PDMS membrane surface.

\subsection{Experimental}

The patterned PDMS membranes are fabricated using interference lithography in patterning process of a thin photoresist layer and followed by imprinting of liquid PDMS. First, 2-3 $\mu \mathrm{m}$ thin layer of positive photoresist AZ 5214E was

\footnotetext{
* ${ }^{1}$ Dusan Pudis, ${ }^{1}$ Daniel Jandura, ${ }^{1}$ Peter Gaso, ${ }^{1}$ Lubos Suslik, ${ }^{2}$ Pavol Hronec, ${ }^{1}$ Ivan Martincek, ${ }^{2}$ Jaroslav Kovac, ${ }^{1}$ Sofia Berezina

${ }^{1}$ Dept. of Physics, University of Zilina, Slovakia

${ }^{2}$ Inst. of Electronics and Photonics, Slovak University of Technology, Bratislava, Slovakia

E mail: pudis@fyzika.uniza.sk
} 
spin coated on GaAs (100) substrate using SPIN 150 coater. The photoresist layer was patterned by interference lithography in Mach-Zehnder configuration using multiple exposure process [9]. The interference optical field is formed by the interference of two coherent beams of Toptica laser operating at wavelength of $403 \mathrm{~nm}$. Interference optical field exposed the thin photoresists layer. Its period can be simply adjusted by anlge of incident beams. For this experiment the angle was 48 degree, corresponding to wavelength of $495 \mathrm{~nm}$. The 10x expander was used for exposure homogeneity improving in the exposed area with diameter of $5 \mathrm{~mm}$ and exposure intensity of $25 \mathrm{~mW} / \mathrm{cm}^{2}$ in both laser beams.

2D PhC structure of square symmetry was achieved by double exposure process, with a sample perpendicular rotation between exposures [10]. After exposure, the sample was developed in $\mathrm{AZ} 400 \mathrm{~K}$ developer for $10 \mathrm{~s}$ and rinsed in deionized water. Using the interference lithography process we obtained patterned surface in thin photoresist layer in area of $5 \times 5 \mathrm{~mm}^{2}$.

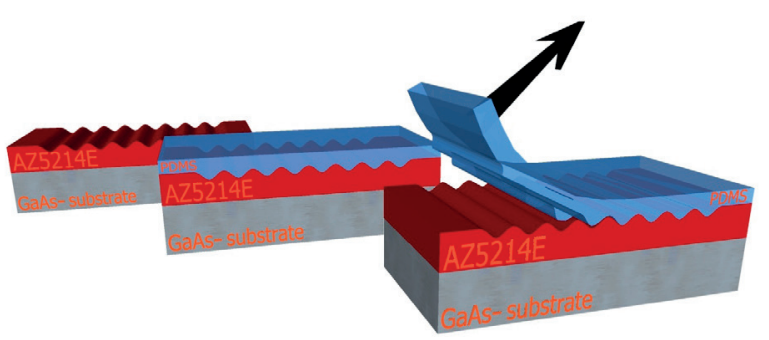

Fig. 1 PDMS membrane fabrication process using imprinting process with patterned positive photoresist AZ5214E deposited on GaAs substrate

In the next process, polydimethylsiloxane layer was deposited. Liquid PDMS was prepared from components of Sylgard 184 elastomer and curing agent at ratio 10:1. For a uniform surface, the $30 \mu \mathrm{m}$ thin PDMS layer was spin coated at $4000 \mathrm{rpm}$ on the patterned photoresist layer. Subsequently, the sample was cured for $45 \mathrm{~min}$ at $75^{\circ} \mathrm{C}$. After PDMS curing, the PDMS membrane was mechanically removed from the sample (Fig. 1). The original patterned photoresist structures and imprinted PDMS membranes were finally investigated in optical microscope and atomic force microscope (AFM).

\subsection{Results and discussion}

Quality of imprinted PDMS membranes as well as original structures prepared in the photoresist layer was analyzed in AFM microscope as is shown in Fig. 2. In Fig. 2a, AFM image of original photoresist master prepared in the thin photoresist layer is shown. Fig. $2 b$ shows surface of imprinted PDMS membrane. Period of the photoresist structure is $495 \mathrm{~nm}$ and imprinted structure in PDMS preserves the photoresist pattern with the same period. Thickness of prepared PDMS membranes is app. $30 \mu \mathrm{m}$. Membrane thickness can be controlled by PDMS viscosity and speed of coating process. Much thinner membranes could be prepared, however, manipulation of thin PDMS membrane is then complicated. More detailed in-depth analysis was investigated from line profiles of AFM analysis taken at diagonal direction of 2D structure (Figs. 2c and 2d). Depth of app. $140 \mathrm{~nm}$ was observed from AFM line profile for the patterned photoresist layer (Fig. 2c) and nearly $100 \mathrm{~nm}$ for imprinted PDMS membrane surface (Fig. 2d).

Such patterned PDMS membrane can be directly applied on optoelectronic devices, e.g. on top of a LED chip or other elements. Such PhC structures have capability to significantly improve their optoelectronic properties as light extraction efficiency and far-field radiation pattern [10].

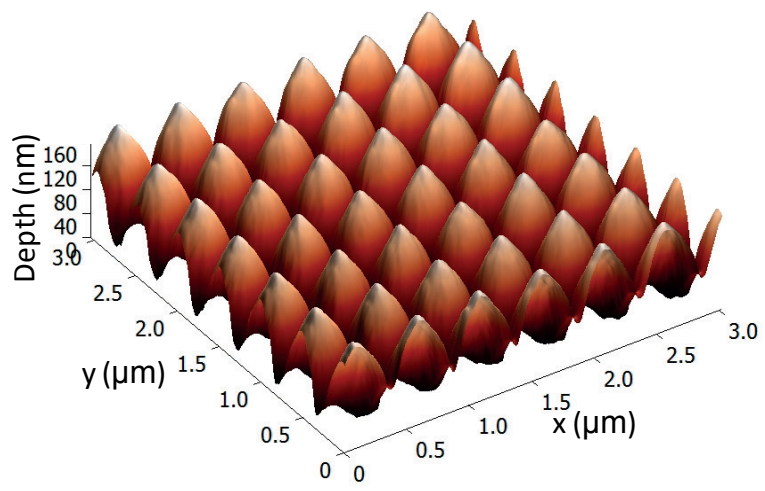

a)

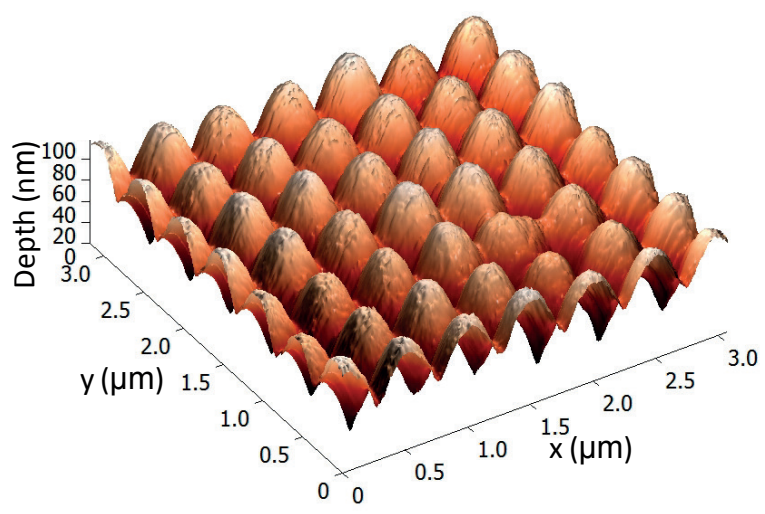

b) 


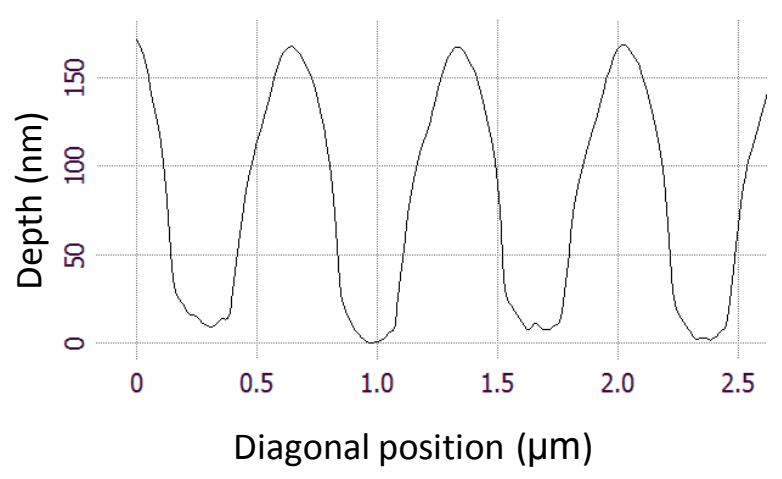

c)

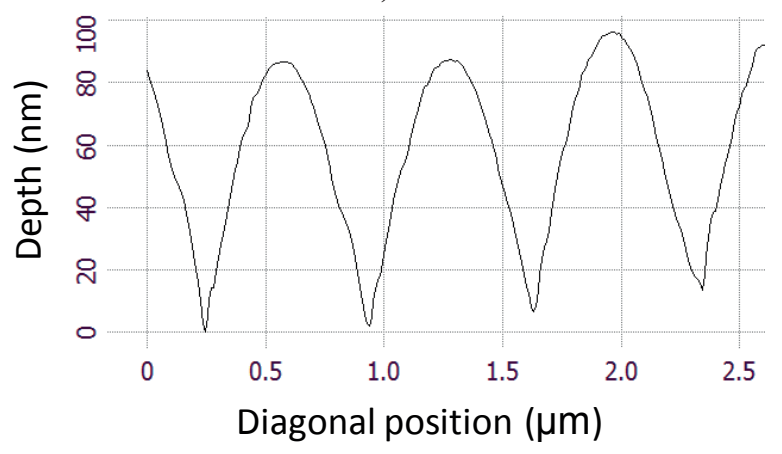

d)

Fig. 2 AFM images of surfaces: a) original patterned photoresist layer and b) imprinted PDMS surface. Line profile of patterned c) photoresist layer and d) imprinted PDMS membrane taken at diagonal direction

\section{PDMS membranes for microptics}

Improving of light divergence from small light emitting sources motivates to fabrication of micro-optic devices. The main idea of this part is to fabricate Fresnel zone plate for improvement of beam divergence from a LED chip using experimental experiences with PDMS membrane fabrication. PDMS is appropriate material on which FZP can be prepared and directly applied in a LED surface. For the FZP fabrication we used direct laser writing (DLW) method for a thin photoresist layer patterning and followed by Al etching process on PDMS membrane surface [11].

FZP is a structure where alternate transparent and opaque zones. These zones are defined by concentric circles with defined radius. Appropriate radius is given so, that the incident light constructively interferes at the focus of FZP. It follows that the arrangement of zones depends on the collimated light wavelength and desired focus distance. Radius of individual circles is then given by following equation

$$
R_{n}=\sqrt{n \lambda f+\frac{n^{2} \lambda^{2}}{4}}
$$

where $n$ is integer describing order of circle, $\lambda$ is wavelength and $f$ is focus distance. With respect to this equation and desired focal length, we prepared FZP on glass and PDMS substrate.

\subsection{Experimental}

Patterning of a thin photoresist layer was achieved by scanning of a focused laser beam on a sample surface. High resolution dual-axes galvanometer mirror system GVSM002 controlled by computer was used for DLW technique. To control scanning system, the LabView program automatically calculates desired radius of FZP circles and applies them to control scanning process of galvanometer mirrors. In arrangement with $10 \mathrm{x}$ objective the scanning range of the system is few hundreds of micrometers and the focused laser spot in the photoresist layer achieves resolution better than $3 \mu \mathrm{m}$ [12].

For resolution optimization and focusing capability, the glass substrate with a thin deposited Al layer was used. In order to create opaque zones on glass, a $50 \mathrm{~nm}$ Al layer was evaporated on the glass surface using K975X turbo-pumped thermal evaporator. Positive photoresist was used as a mask for Al layer patterning (Fig. 3a).

Using $405 \mathrm{~nm}$ laser beam, the sample was irradiated through the objective at desired positions. After exposure, the sample was developed and subsequently wet etched in solution of $\mathrm{H}_{3} \mathrm{PO}_{4}$ and ethanol at the ratio $1: 10$ at $50^{\circ} \mathrm{C}$, where uncovered $\mathrm{Al}$ parts were etched (Fig. 3b). Finally, the remaining photoresist was removed in solvent (Fig 3c).

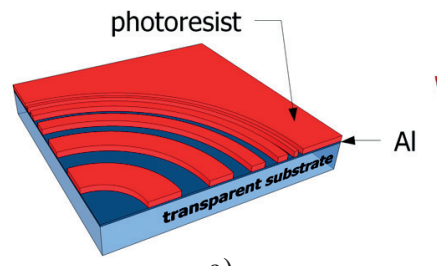

a)

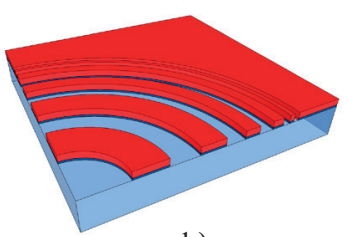

b)

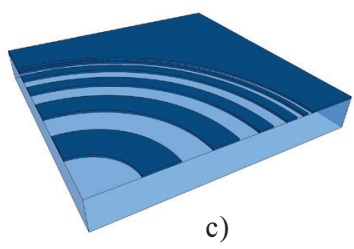

Fig. 3 FZP fabrication process: a) exposition and developing process of thin photoresist layer deposited on Al layer

b) Al layer wet etching c) photoresist removing

Transparent parts of created FZP are shown in optical microscope image in Fig. 4a. Fabricated FZP structure was 
designed for wavelength $\lambda=600 \mathrm{~nm}$ and for focal length $f=10 \mathrm{~mm}$. In order to apply FZP directly on LED chip we prepared FZP on a thin PDMS layer. For uniform surface, the $30 \mu \mathrm{m}$ thin PDMS layer was spin coated at $4000 \mathrm{rpm}$ on a glass substrate. Subsequently, the sample was cured for $45 \mathrm{~min}$ at $75^{\circ} \mathrm{C}$. FZP based on PDMS layer was patterned by the same lithography process using thin positive photoresist layer $(3 \mu \mathrm{m})$ followed by exposure using DLW technique. The FZP prepared on PDMS membrane is shown in Fig. 4b.

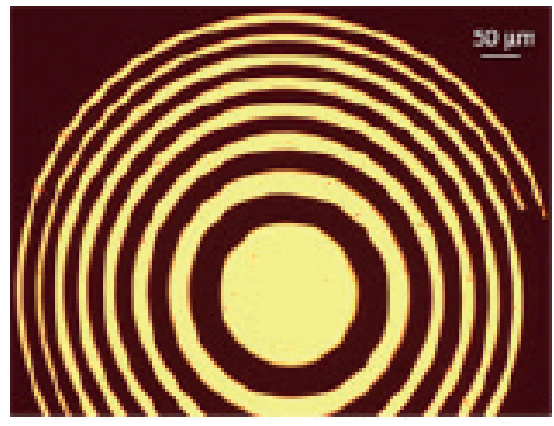

a)

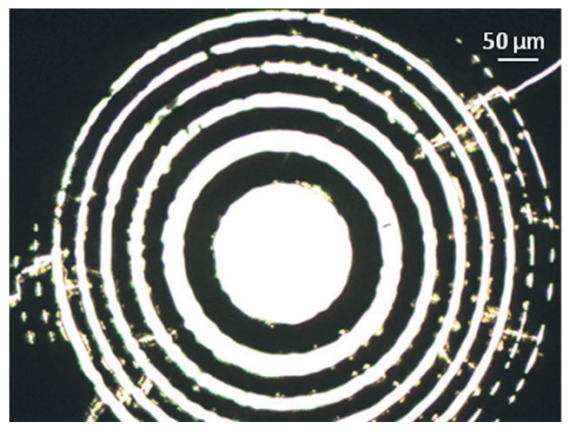

b)

Fig. 4 Optical microscope image of FZP structure prepared on a) glass and b) PDMS surface

The focal length of prepared FZP was measured in simple experimental arrangement LED source-FZP-CCD camera and calculated using standard lens equation. The measured focal length was approximately $9.5 \mathrm{~mm}$, which nearly corresponds to the designed FZP with $f=10 \mathrm{~mm}$. The real image of orange LED source with central wavelength $\lambda=612 \mathrm{~nm}$ using prepared FZP is shown in Fig. 5. Due to a wide spectral range of LED source the real image is not perfectly contrasted, because the FZP focal length shows dependence on the used wavelength. The orientation of the image is reversed in comparison with original object as is shown in inset figure in Fig. 5, which fulfils the basic optical imaging principle and lens equation. We conclude that FZP based on PDMS can be directly applied on the LED surface and can lead to modification of beam shape.

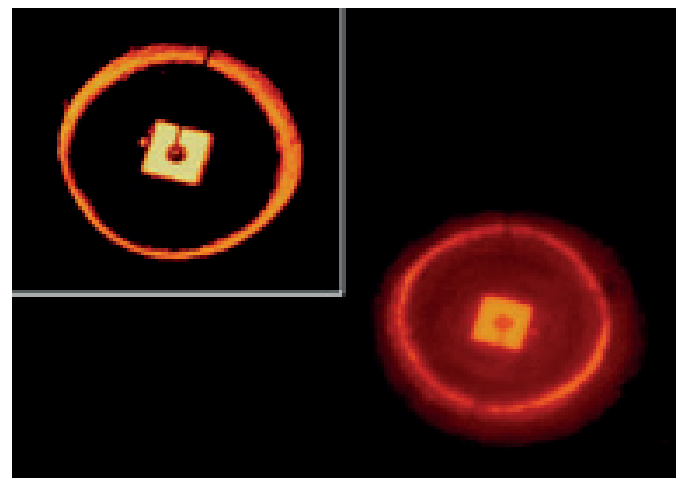

Fig. 5 Real LED image after imaging using FZP and original object (inset figure)

\section{Surface-relief fiber bragg grating in PDMS}

In this part, we describe a new fabrication method for SR FBG based on PDMS waveguide fabricated by combination of interference lithography process and imprinting technique. PDMS shows unique elastic properties allowing elongation for more than $100 \%$, which may be attractive in tunable optic devices based on FBG [13 and 14].

\subsection{Experimental}

For the fabrication of SR FBG in the PDMS layer, the combination of interference lithography and imprinting technique with liquid PDMS was used. Individual steps of the whole process are described in Fig. 6. The first step is hand made fiber drawing process from positive photoresist AZ5214E that will finally form a core of waveguide (Fig. 6a). It was necessary to achieve appropriate fiber diameter in the range 10-100 $\mu \mathrm{m}$. In the next step, the prepared fiber was placed on a PDMS layer (Fig. 6b). For fabrication of the PDMS layer, we used PDMS Sylgard 184. It was prepared in the same way as described in PDMS membrane fabrication. It is necessary to achieve partial curing of the PDMS at temperature of $60{ }^{\circ} \mathrm{C}$ for about 20 minutes which ensures appropriate viscosity. If a photoresist fiber is placed on such a PDMS layer, the fiber is slightly immersed. Subsequently, the photoresist fiber is exposed by one dimensional interference optical field (Fig. 6c). Interference workplace based on Mach-Zehnder configuration was used to create onedimensional interference pattern. Exposed structure photoresist fiber - PDMS layer was developed in AZ400 K developer to remove exposing parts of photoresist and rinsed in deionised water. One-dimensional periodic surface structure with length of app. $1 \mathrm{~mm}$ was patterned on the photoresist fiber surface with normal orientation to the fiber axis (Fig. 6d). 

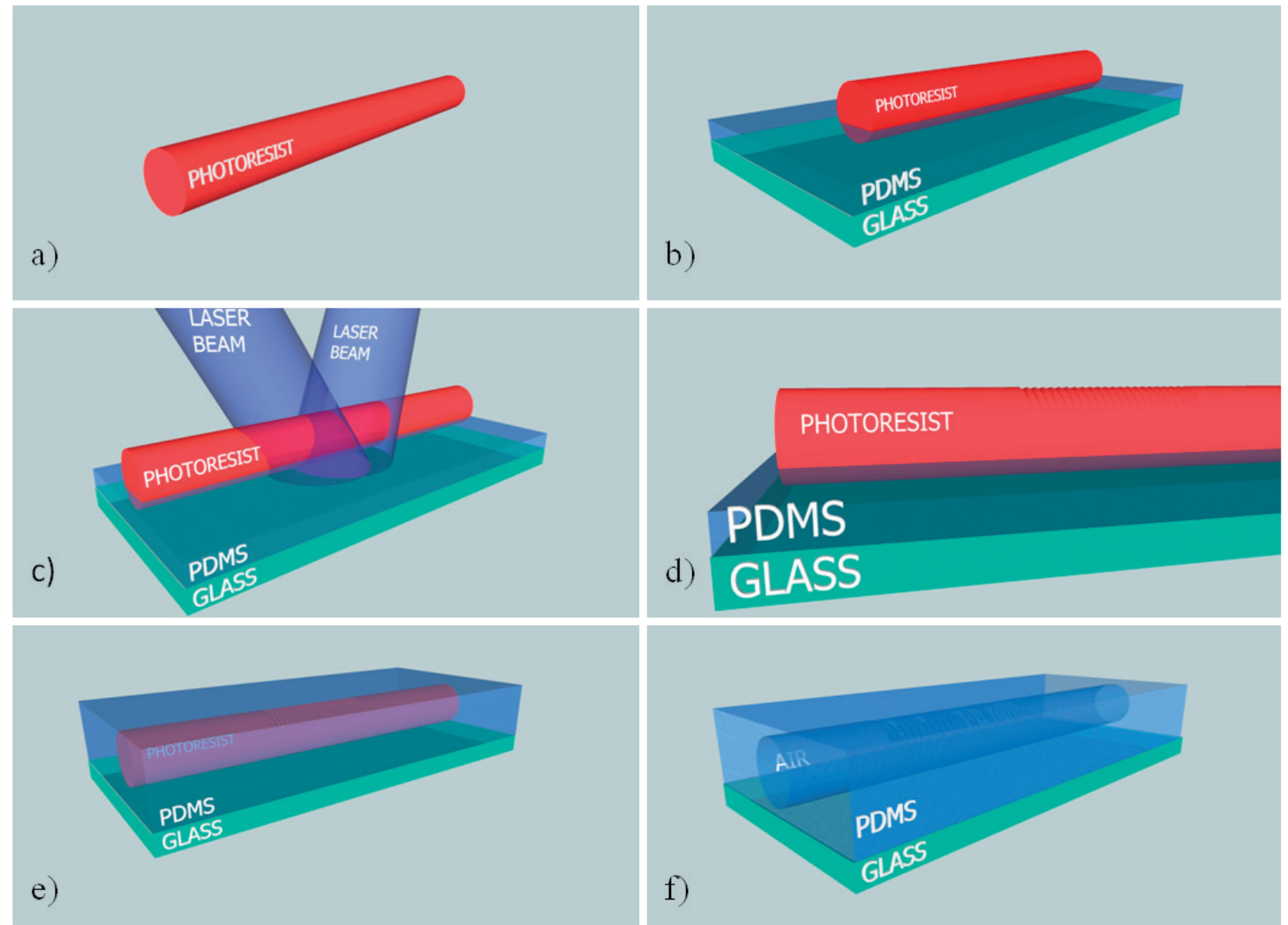

Fig. 6 Schema of SR-FBG fabrication process in PDMS layer

The patterned photoresist fiber was covered with an additional layer of PDMS in order to form a waveguide core (Fig. 6e). Onedimensional structure from the photoresist fiber was imprinted into the covered PDMS layer. After curing PDMS in the next step, photoresist was removed in Microposit Solvent EC 11 in a long time process (app. 24 hours) (Fig. 6f). By this process, the SR-FBG is obtained in the PDMS material.

\subsection{Results and discussion}

After removing the photoresist from PDMS waveguide, we obtained air core waveguide structure in PDMS material with SR-FBG. Fig. 7 shows microscope image of SR-FBG patterned in thin photoresist fiber placed on PDMS layer.

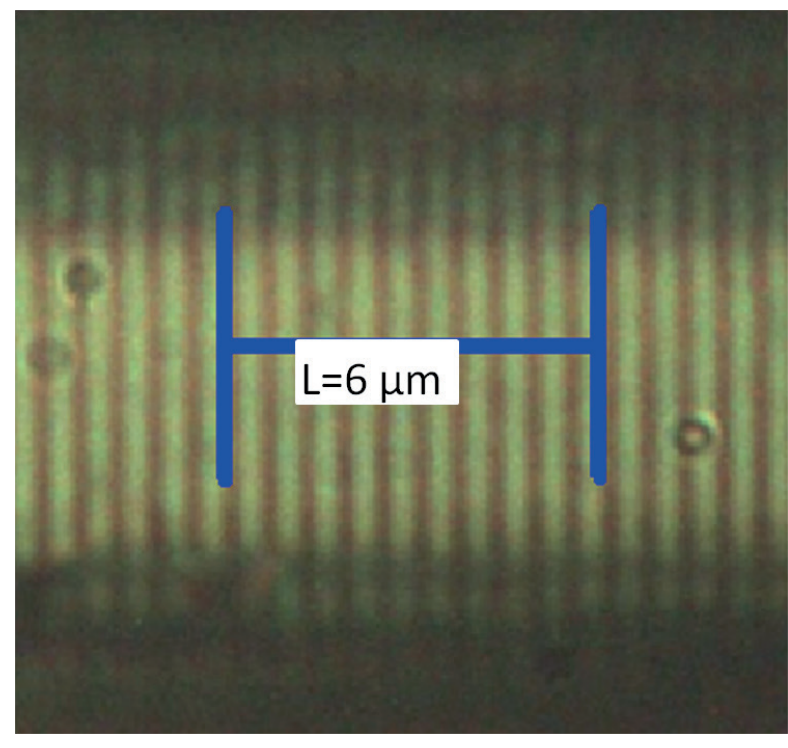

Fig.7 Optical microscope image of prepared SR-FBG on surface of photoresist fiber 
The prepared air core waveguide is not appropriate for further low-loss guiding application. Then the core was filled with material which refractive index greater than PDMS cladding $(n>1.45)$. For this purpose, a different mixture of PDMS was used. Such prepared PDMS structure can be used in waveguide applications for light filtration in specified region.

\section{Conclusion}

We presented few patterning techniques leading to fabrication of PDMS based micro- and photonic structures. We prepared PDMS membranes with 2D PhC patterned surface of period $495 \mathrm{~nm}$ and depth of $100 \mathrm{~nm}$ using interference lithography and imprinting technique. Such membranes can be simply applied on different optoelectronic device surfaces and could modify final optical properties.

We also described fabrication of PDMS membranes for micro-optics. We presented fabrication of Fresnel zone plate structure prepared on PDMS substrates using direct laser writing technique. We favor this FZP prepared on PDMS substrate for direct application on LED chips, which may be attractive for output beam collimation.
We presented new fabrication method of SR-FBG waveguide prepared in PDMS layer. Waveguide structure with SR-FBG was prepared in PDMS with period of $600 \mathrm{~nm}$. For patterning of Bragg grating in fiber surface, the interference lithography was used in combination with imprinting technique. Fabricated SR-FBG in PDMS can be used for application in waveguide optics and optoelectronics.

Mechanical and optical properties of PDMS favor this material for application in optic and optoelectronic devices. Variability of presented techniques opens wide possibilities to form submicrometer periodic structures in PDMS based devices.

\section{Acknowledgement}

This work was supported by the Slovak National Grant Agency under the projects No. VEGA 1/1058/11 and 1/0528/12 and the Slovak Research and Development Agency under the project No. APVV 0395 12. The authors wish to thank for the support to the R\&D operational program Centre of excellence of power electronics systems and materials for their components II. No. OPVaV-2009/2.1/02-SORO, ITMS 26220120046 funded by European regional development fund (ERDF).

\section{References}

[1] JOANNOPOUlOS, J. D., MEADE, R. D., WINN, J. N.: Photonic Crystals - Molding the Flow of Light, Princeton University Press, Princeton 1995.

[2] LOURTIOZ, J. M.: Photonic Crystals - Towards Nanoscale Photonic Devices, Springer-Verlag Berlin Heidelberg, Berlin 2008.

[3] KIM, S. H., LEE, K. D., KIM, J. Y., KWON, M. K., PARK, S. J.: Nanotechnology 18, 2007, p. 055306.

[4] ALTUG, H., VUCKOVIC, J.: Opt. Express 13, 2005, pp. 8819-8828.

[5] LONCAR, M., NEDElJKOVIC, D., DOLL, T., VUCKOVIC, J., SCHERER, A., PEARSALl, T. P.: Appl. Phys. Lett. 77, 2000, pp. 1937-1939.

[6] FUJII, T., GAO, Y., SHARMA, R., HU, E. L., DENBAARS, S. P., NAKAMURA, S.: Appl. Phys. Lett. 84, 2004, pp. 855-7.

[7] KRAUSS, T. F.: Phys. Stat. Sol. 197, 2003, pp. 688-702.

[8] VAlOUCH, S., SIEBER, H., KeTTlitZ, S., ESCHENBAUM, C., HOLlENBACH, U., LEMMER, U.: Opt. Express 20, 2012, pp. 28855-28861.

[9] SKRINIAROVA, J., PUDIS, D., MARTINCEK, I., KOVAC, J., TARJANYI, N., VESELY, M., TUREK, I.: Microelectron. J. 38, 2007, pp. 746-749.

[10] PUDIS, D., SUSLIK, L., SKRINIAROVA, J., KOVAC, J., MARTINCEK, I., KOVAC JR., J., HASCIK, S., KUBICOVA, I., NOVAK, J., VESELY, M.: Optics and Laser Technol. 43, 2011, pp. 917-921.

[11] YANG, R., TANG, W., HAO, Y.: Opt. Express 19, 2011, pp. 12348-12355.

[12] PUDIS, D., KUBICOVA, I., GASO, P., JANDURA D.: Technolog 1, 2012, pp. 13-15.

[13] MELTZ, G., MOREY, W. W., GLENN, W. H.: Opt. Lett., Vol. 14, 1989, pp. 823-825.

[14] OTHONOS, A., KALLI, K.: Fiber Bragg Gratings: Fundamentals and Applications in Telecommunications and Sensing, Artech House, Boston, 1999. 